\title{
Humanoid Robot Programming for the Assistance of an Artistic Performance
}

\author{
Adrián Antonio Castañeda Galván ${ }^{1}$, Álvaro Anzueto Ríos², \\ Mariana Elizabeth Montealegre Rodríguez ${ }^{2}$ \\ ${ }^{1}$ Instituto Politécnico Nacional, UPIITA, Electrónica, CDMX, \\ Mexico \\ ${ }^{2}$ Instituto Politécnico Nacional, UPIITA, Biónica, CDMX, \\ Mexico
}

acastanedag@ipn.mx, aanzuetor@ipn.mx,mariana.elizabeth13@yahoo.com.mx

\begin{abstract}
In this paper the methodology of programming a Darwin-Op® humanoid robot for the assistance of an artistic performance is shown. A performance is composed of elements that express emotions and feelings in people, fulfilling a series of movements defined by sequentially angles, lengths of the limbs and joints of the body. A biomechanical analysis was done for a dimensional relationship between humans and humanoid robot movements and allow a choreographic environment, which are sequences of basic movements accompanied by music in sync. The vision system Motive Tracker OptiTrack® and costume withmarkers to analyze the graphs, positions and trajectories of a skeleton in a three dimensional space generated for each routine, to obtain the angular information required was used. The extracted angles represent a sequence of movement and are placed on a table that is used to program the DarwinOp ${ }^{\circledR}$ humanoid robot with Roboplus $®$, this program to take care of mimicking the sequence performed by humans. The positions of the servo motors in each static pose was plotted and compared to those generated with the test subject. The degree of error is at its peak $5 \%$, demonstrating that the humanoid robot has a mimicry of human movements. The interaction with the user is carried out by processing voice commands to call specific routines programmed into the humanoid robot.
\end{abstract}

Keywords: Humanoid robot, Darwin-Op®, performance, biomechanical analysis.

\section{Introduction}

Man has sought to develop robots to increase its data processing capacity to interact with the environment and especially trying to mimic human anatomy [1], from which arise the so-called humanoid robots that have bipedal walking [2]. Nowadays, the advancement of robotics has become a contribution to the development of society in 
different fields such as medicine, industry, entertainment, among others. In the near future, the use of humanoid robots will increase in modern societies and will have applications in areas of entertainment with social interaction and education.

In the field of this work are among others the project called "Guided gestural of a humanoid robot using a Kinect sensor", where a biomechanical model is developed by software Skeleton tracking of Kinect, which allows tracking of the body of a user in real time and applies the model in a humanoid robot Bioloid with implementing firmware control of the actuators of the robot and the inverse kinematics of the arms [3]."Environment to choreograph movements on a Humanoid Robot" It's application presents an environment for modeling choreographies of movement for humanoid robot Robonova. This environment allows users to graphically model in the robotic platform and formally validate the sequences of movements of the robot (choreography) and automatically generate the implementation associated with each choreography in robot programming language, called roboBASIC [4]. The project "Humanizing Robot Dance Movements" captured the human movement to dance samba and programmed into the humanoid robot NAO with the robotic simulator SimSpark [5].

The above projects use programs and platforms that give the programmer the movement data the human being, emphasizing the use of the Software Model-Driven (DSDM). In our case all the analysis and modeling was developed with a view to the Darwin-Op® robot is part of an artistic performance that has an interaction with humans through voice commands for each routine and thereby give more work guideline for social interaction and teaching dance steps for children

\section{Materials and Methods}

For the development of this project 34 infrared decoys were made, the Motive Tracker® program for recording movement, Camtasia ${ }^{\circledR}$ and Kinovea ${ }^{\circledR}$ was used to analyze video pictures, RoboPlus ${ }^{\circledR}$ and VNC Viewer ${ }^{\circledR}$ to program routines in the robot, Audacity to synchronize music tracks and DarwinOp ${ }^{\circledR}$ platform to run the preprocessed models.

The first step consists in selecting a genre of music, in order to observe the movement capability of the DarwinOp® humanoid robot and its direct relationship with humans. Every musical genre was obtained basic information such as speed of movement, characteristic steps of the musical genre and the bit (musical time) style itself, which allows the synchronization of steps to music. Subsequently, a static set of movements, which together correspond to a choreographed routine identified and parameterized angles that can perform DarwinOp® robot with angles that are required to execute the routine choreography generated; audio tracks together with the corresponding Audacity program performance and execution times of the robot were made. To do this the following methodology was applied:

\subsection{Registration of Movement in the Vision System Motive Tracker ${ }^{\circledR}$}

34 infrared decoys of fluorescent markers were constructed to create a skeleton movement called Baseline (34) for recording and analysis of the lower body and upper 
body in the vision Motive Tracker® system as shown in Figure 1. Once placed markers in the body as indicated in the program the choreography for the performance was executed and stored in the sessions generated by the vision system.

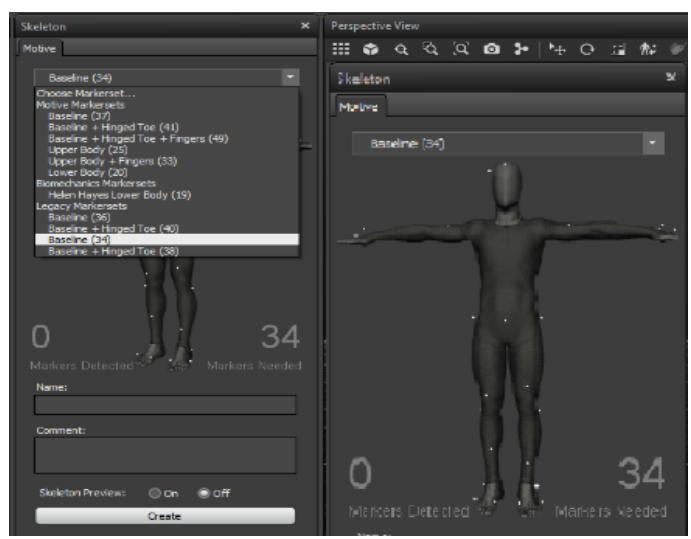

Fig. 1. Motive Tracker baseline 34.

\subsection{Extraction of Graphics and Videos Generated in the Vision System}

The Motive Tracker® vision system works in three dimensional space, so there are 34 graphs to the axis "x", 34 for the "y" axis and 34 to the axis "z", which indicate the path traveled by each marker on each axis in the performance. Thus, keep sessions in which through its data makes a reconstruction of 3D motion (Figure 2). but it cannot export the video to a processable format by this reason Camtasia program was used, because it allow us record the pc screen and in this manner create a video with the routine performance previously captured.

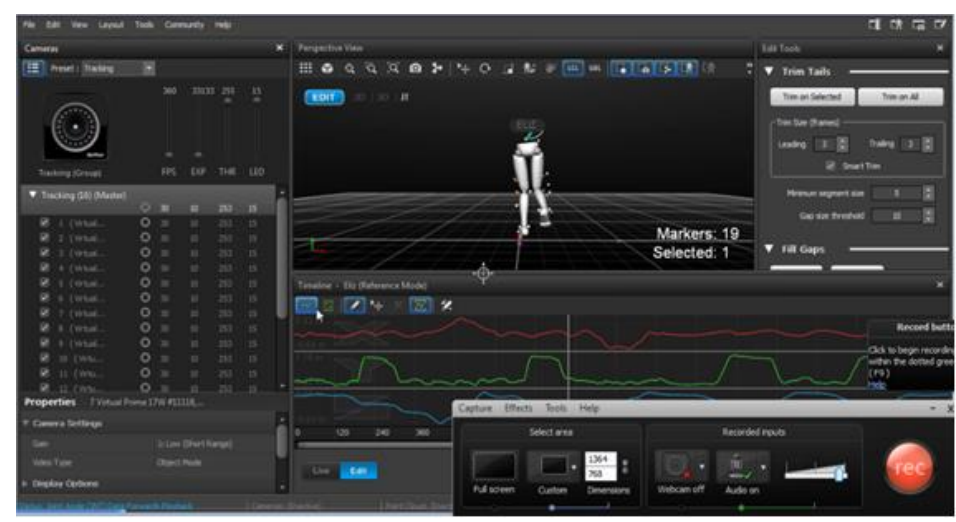

Fig. 2.Video and graphics extraction with Camtasia.

The vision system Motive Tracker® requires controlled lighting conditions, as well as reflective decoys placed in the suit that includes this system to generate the skeleton. 
Subsequently graphics and video extraction for each marker for each movement routine are made with Camtasia ${ }^{\circledR}$. Three angular analyzes were performed corresponding to the lateral, superior and frontal sides of view, thus for obtain the articular movements of each 20 degrees of freedom (DOF) for the robot Figure 3

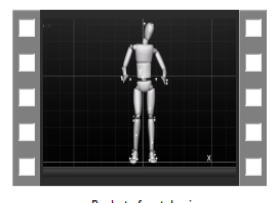

Bachata frontal.avi

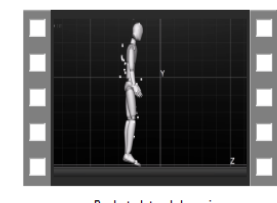

Bachata lateral der.avi

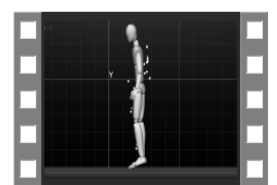

Bachata lateral iq.avi

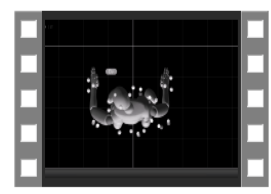

Bachata superior.avi

Fig. 3. Sides of view extracted from the vision system. (screenshot in Spanish).

\subsection{Angular Movement Analysis with Kinovea ${ }^{\circledR}$}

Every video of lateral, frontal and superior sides of view of each movement routines obtained with Camtasia ${ }^{\circledR}$ and Motive Tracker ${ }^{\circledR}$ are analyzed with Kinovea ${ }^{\circledR}$ to obtain the angular position of the 20 angles corresponding to the 20 DOF of the actuators DarwinOp® robot, see Figure 4.

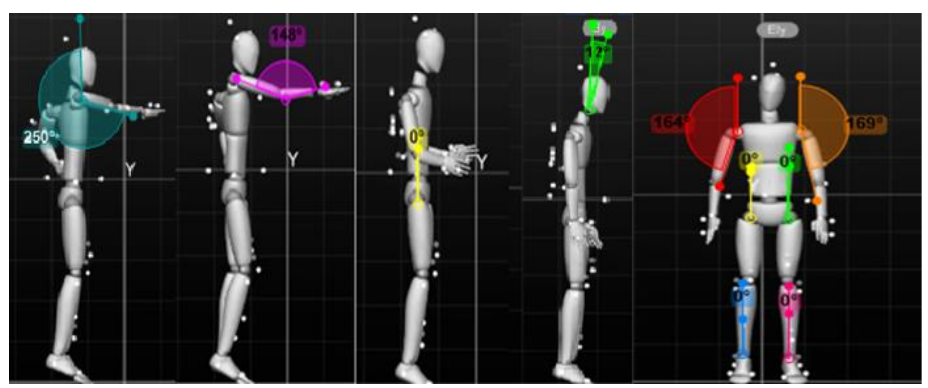

Fig. 4. Angular Analysis with Kinovea ${ }^{\circledR}$.

\subsection{Analog to Digital Angular Conversions to Drive Darwinop® Servo Motors}

The motors 20 DarwinOP robot are digital servomotors Dynamixel MX28 which are configured to generate movements between $0^{\circ}$ and $360^{\circ}$ with numbers between 0 and 4095 respectively.

Each servomotor due to its location in the robot has different minimum and maximum limits to generate joint movements. These limits are programmed in a card called internal Control CMX730.

Because of this, it is necessary to convert all angular movements tables routines to digital values required by servomotors. Each frame of the video obtained with Kinovea ${ }^{\circledR}$ was processed in an Excel ${ }^{\circledR}$ program to convert all angles to digital values that the robot can obey as particular position. The equation is shown below.

$$
\begin{aligned}
& \text { Ángulo } \\
& =\operatorname{INDICE}\left(\operatorname{Servo}_{\#}\left(A_{c}: A_{f}\right), \operatorname{COINCIDIR}\left(\operatorname{Celda}_{c o m p}, \operatorname{Servo}_{\#}\left(A_{c}: A_{f}\right),-1\right), 2\right) \ldots
\end{aligned}
$$




$$
\begin{aligned}
& R E S 1=\text { Coincidir }\left(\text { Celd }_{\text {comp }}, \operatorname{Servo}_{\#}\left(A_{c}: A_{f}\right),-1\right) \\
& \text { POS }_{\#}=I F\left(\left(\frac{4096 * \text { Ángulo }}{360}\right.\right. \\
& \left.\leq \text { ServoMin }_{\#}\right), \text { ServoMin }_{\#}, \text { IF }\left(\left(\frac{4096 * \text { Ángulo }}{360}\right.\right. \\
& \left.\left.\left.\geq \operatorname{ServoMax}_{\#}\right), \operatorname{ServoMax}_{\#,}\left(\frac{4096 * \text { Ángulo }}{360}\right)\right)\right) \ldots
\end{aligned}
$$

Subsequently a data base with the digital values that needs the robot of each movement are generated.

\subsection{Humanoid Robot Programming and Synchronization to a Music Track}

To load the robot movements the network cable connected from the computer terminal to terminal DarwinOP® and Ultra VNC Viewer ${ }^{\circledR}$ program was implemented to allow us RoboPlus ${ }^{\circledR}$ run from the command console and the robot from the computer. This is necessary to change the servo motors digital values Figure 5.

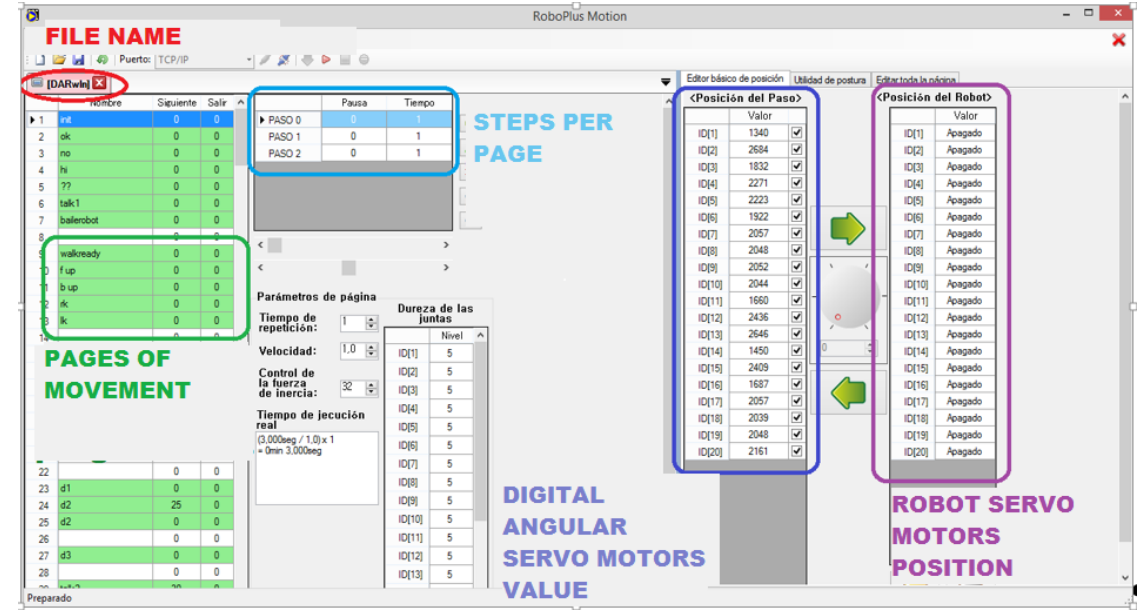

Fig. 5. RoboPlus ${ }^{\circledR}$ screen to program humanoid robot.

Once in the program, pages are created to send for movements, each page can have 7 steps and each step biomechanical models are programmed [6] obtained from the analysis of data in Kinovea ${ }^{\circledR}$ and the database generated with the values digital for every servo motor.

From the database in Excel can be copied directly to the digital values of the 20 servomotors and then press the right arrow to allow engines put the desired position, thus can verify the performance to be programming step by step. 
It is important to note that not only is mark positions in sequence on the robot, you should use the established parameters and correct the balance of the robot, using information known as well as the information obtained by the human who performs the same sequence of movements.

After the programming of a complete routine, the movements generated to the corresponding track are synchronized. The process of this stage starts running track and the sequence of movements created at the same time, it should be noted that movements go with the music time, if at any point the movements are faster or slower than the music must be corrected speed steps, also, if by changing the amount of movement speed is less than the length of the track, should add new pages, new steps for the missing track time.

\subsection{Wireless Communication, Voice Processing and Commands}

Voice processing was performed on another computer with Linux operating system Ubuntu 12.04, as other versions are not compatible or generate errors with libraries for speech processing and computer robot uses Linux 9.04 or 10.1 .

The voice processing program loads the necessary libraries containing phonemes and parcels to initialize the voice recognition feature, check that the audio system work properly and requirements for the microphone are made. Because the total of routines scheduled for this performance are three the number of commands corresponding to these routines, identified by orders "one, two, three".

In order to make a WiFi wireless communication and TCP / IP protocol module is used because this channel ensures that all data to be transmitted arrive in order, complete and unabridged. It requires therefore a program for server and for client one. The voice processing program is the server where the routine is executed in the robot, it is the customer. So the program on the server data acquisition (phonemes) compared with phonemes is established, identifies the order of appearance and the probability of occurrence and finally the program sends the robot routine to be executed.

\section{$3 \quad$ Results}

The performance consists of three scheduled routines, gender chosen for the routine one was pop music, with the song "La Macarena" by Los del Rio, this song consists of predefined steps that were used as a basis for the performance of the robot, this routine lasts one minute with thirty four seconds, this was scheduled starting on page number 98 and ending on page number 117, which gives us a total of 20 pages programmed, each of these pages contains a number of steps between one and seven, which modify the 20 servomotors, moving from one position to another, with the programmed sequence and the required speed.

For routine two, music genre was "bachata" with a remix of songs titled "Obra Maestra" and "Amigo" from Romeo Santos; this genre takes steps base with hip movements, arm movements are titled with cadences and steps bases of this dance, like the so called "arrastra" which is to drag a foot, this routine has a duration of one minute thirty six seconds is scheduled starting at the page number 136 and ending at the page number 162 , which gives us a total of 27 pages programmed. 
In the routine three, the chosen genre was disco music in English with the song "YMCA" by the Village People, 1978. This song was chosen because it is preset to dance steps, lasts one minute and seventeen seconds, it was scheduled starting on page number 190 and ending at the page number 214, which gives us a total of 25 pages programmed. Evidence of this can be seen in Figure 6.

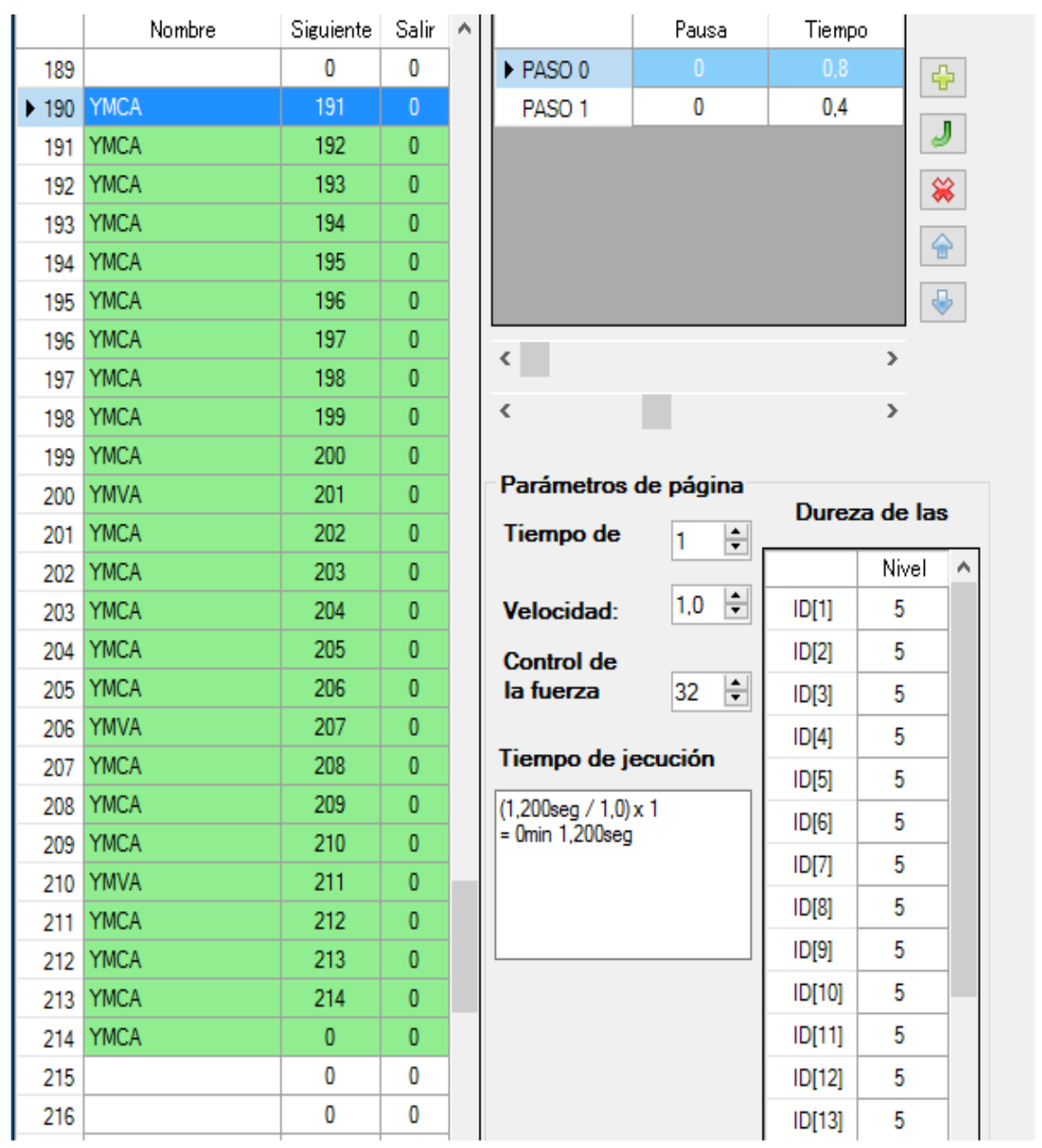

Fig. 6. Pages programmed in routine 3. (Screenshot in Spanish).

For the three established routines have 72 pages of programmed movement, all with about 7 steps per page and 20 digital values for each servomotor, which gives us a total of 504 steps per program and a total of 10,080 angles.

The 10,080 angles are in a database generated in the Excel program, each step has angular positions and the digital values of every movement for each of the 20 servomotors of the robot. Thus for routine 1 we have 20 pages with 7 steps per page giving a total of 140 steps. See Figure 7. 


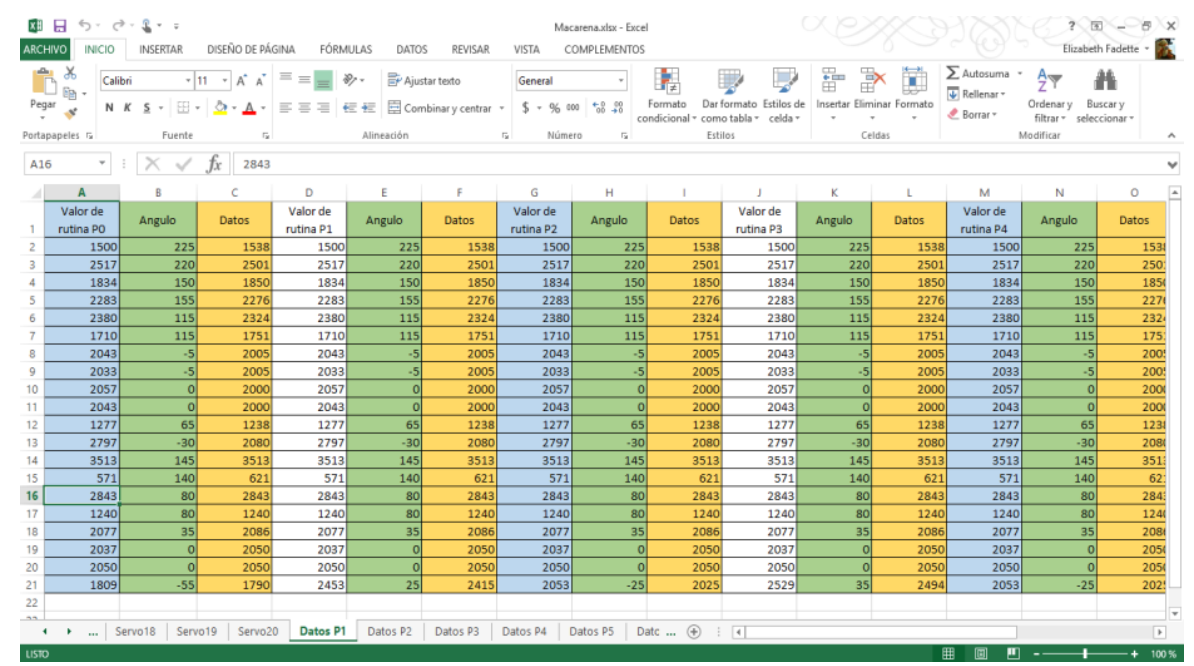

Fig. 7. Excel book with angular values (screenshot in Spanish).

Each step has an image and a table for angles of 20 servomotors, obtained from 3D videos of the lateral, frontal and top views. Of the 504 steps were chosen at random 51 angles and 1020 steps were chosen and read with RoboPlus program and compared with the value angle of the table in the database, as shown in Figure 8.

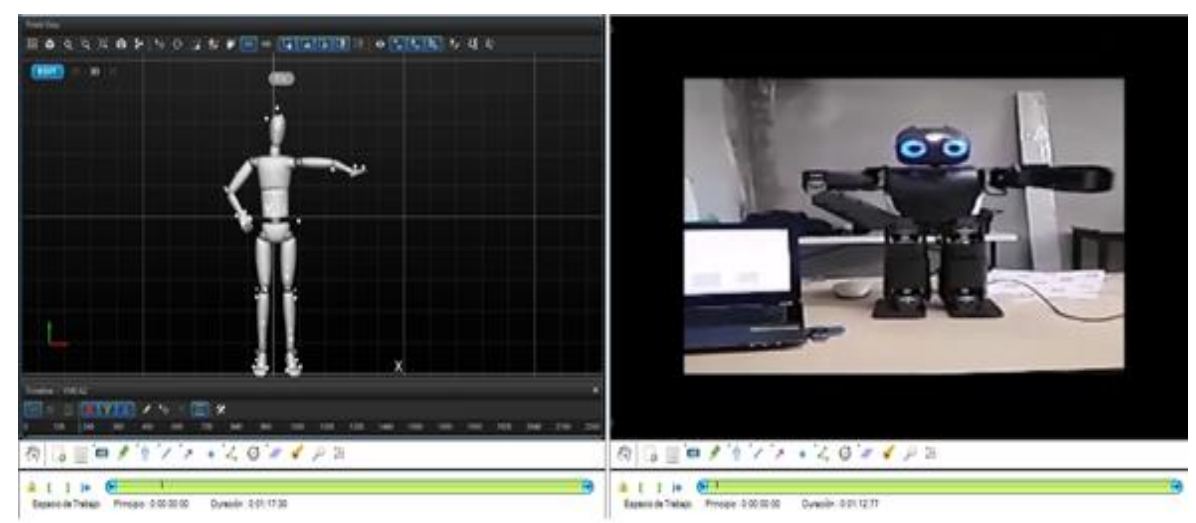

Fig. 8. Angles comparison.

Each motor in the robot has an ARM 32bit microcontroller, which contains settings in PID control to reach the position (Figure 9), so when converting the digital value read from the robot to the angle and compare it with the data table gave us a 5\% error in 100 angles off 1020 ; i.e., $9.8 \%$ of the total sample has a maximum error of $5 \%$ and only in the extremities were the robot makes mayor effort.

For each routine robot video recordings were made and analyzed in the Kinovea ${ }^{\circledR}$ program, which allows us to track a specific point; in this case, a point is placed in a similar position, where the test subject have a marker, the trajectory generated in the 
software is similar to the graph given by the Motive Tracker, as shown in Figure 10 which guarantees that the error rate is $5 \%$.

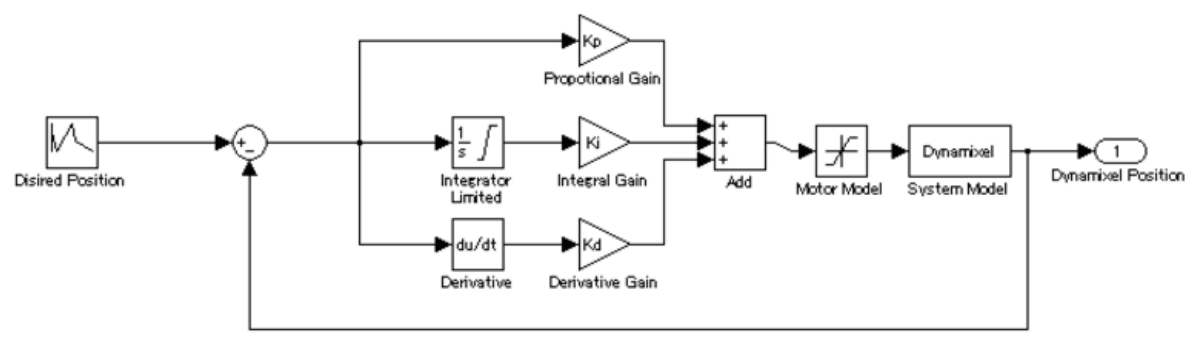

$$
\begin{aligned}
& \mathrm{K}_{\mathrm{p}}=\mathrm{P} \text { Gain } / 8 \\
& \mathrm{~K}_{\mathrm{i}}=\mathrm{I} \text { Gain } * 1000 / 2048 \\
& \mathrm{~K}_{\mathrm{d}}=\mathrm{D} \text { Gain } * 4 / 1000
\end{aligned}
$$

Fig. 9. PID Dynamixel motor control model.

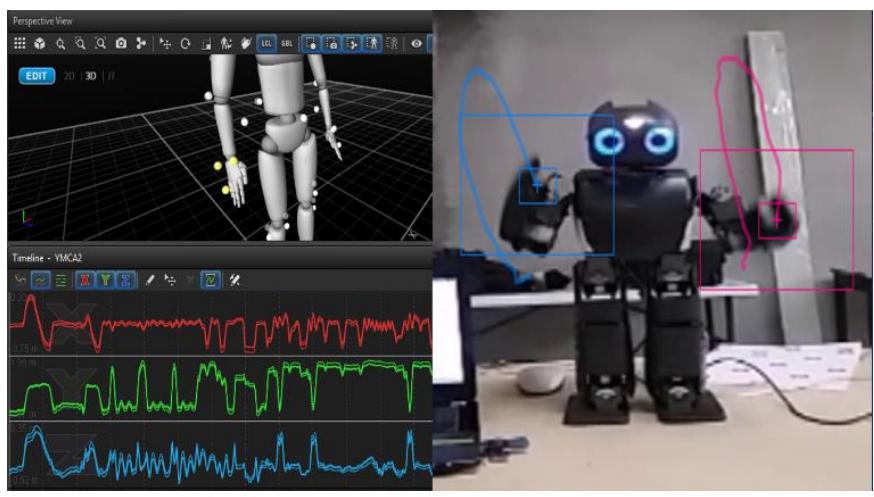

Fig. 10. Graphic comparison.

At this stage it can be seen the visual comparison of some positions, a specific routine that checks to similarity between the performance played by the DrawinOp® ${ }^{\circledR}$ humanoid robot and human.

Acknowledgements. The authors acknowledge the support UPIITAIPN and Dr. Alberto Juarez Luviano for the use of Motive Tracker vision system.

\section{References}

1. Sánchez, M. B.: Diseño y Construcción de un robot humanoide. Escola Universitaria d'Enginyeria Técnica Industrial de Barcelona, Universitat Politécnica de Catalunya, Barcelona (2011)

2. Ambriz, C. F., Arreguín, C. A., Ledesma, J. R.: Congreso Interdisciplinario de Cuerpos Académicos. Ciencias de la Ingeniería y Tecnología, Handbook T-VI, Volumen VI, ECORFAN@ 11 (2014) 
3. Pfeiffer, S.: Guiado gestual de un robot humanoide mediante un sensor Kinect. Facultat D’Informática de Barcelona (2011)

4. Inglés, J., Verdú, R., Vicente, C.: Entorno para Coreografiar Movimientos en un Robot Humanoide. Escuela Técnica Superior de Ingeniería de Telecomunicación, División de Sistemas e Ingeniería Electrónica (DSIE) (2009)

5. Quigley, M., Gerkey, B., Conley, K., Faust, J., Foote, T., Leibs, J.: ROS: an open-source Robot Operating System. In: ICRA workshop on open source software (2009)

6. Kinovea.: http://www.kinovea.org (2016)

7. Izquierdo, M.: Biomecánica y bases neuromusculares de la actividad física y el deporte. Editorial médica panamericana, Buenos Aires Madrid (2008)

8. Sousa, P.: Humanizing Robot Dance Movements, Faculdade de Engenharia da Universidade do Porto (2011)

9. Sullivan, K., Luke, S., Ziparo, V. A.: Hierarchical learning from demonstration on humanoid robots. In: Proceedings of Humanoid Robots Learning from Human Interaction Workshop (2010) 